\title{
Inferior vena cava-right atrial junction stenosis requiring a multidisciplinary approach to resection and reconstruction
}

\author{
Shane P. Smith, MD, ${ }^{a}$ Caleb G. Van Essen, MD, ${ }^{a}$ Adam H. Zivin, MD, ${ }^{b}$ P. Gregory Hayes, MD, ${ }^{b}$ \\ Evan S. Ong, MD, ${ }^{c}$ and Eric J. Lehr, MD, PhD, ${ }^{b}$ Seattle, Wash
}

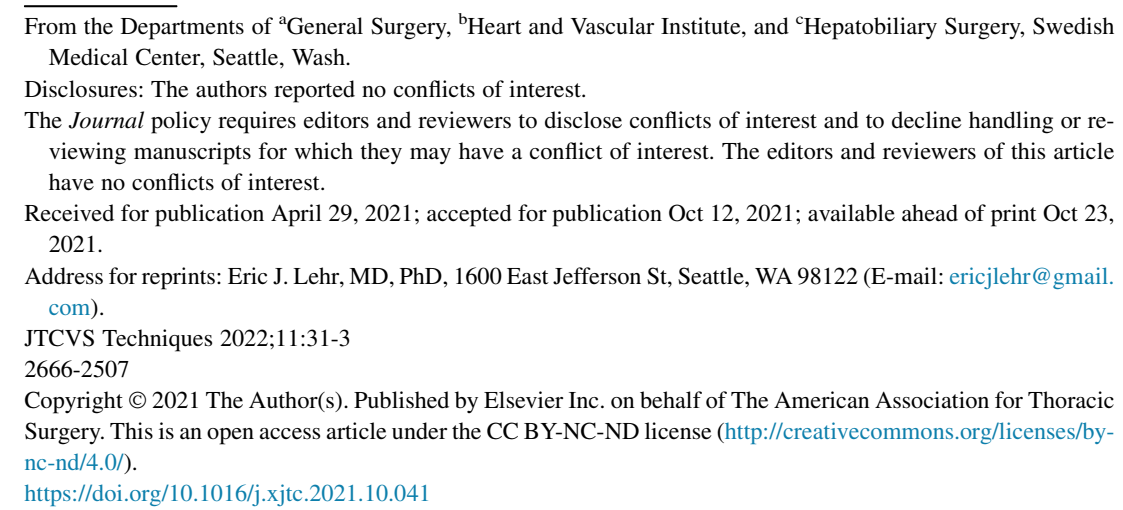

Video clip is available online.

A 28-year-old female patient presented with 4 months of vague abdominal pain and distension. She had a dualchamber automatic implantable cardioverter-defibrillator (AICD) with right atrial and right ventricular leads for prolonged QT syndrome since age 14 years. She was also 20 months postpartum from an uncomplicated pregnancy. Initial workup revealed ascites, and portal hypertension was diagnosed. The was no laboratory concern for intrinsic liver disease, and hypercoagulable and oncologic evaluations were negative (Video 1).

Venography demonstrated accelerated blood flow and a large pressure gradient of $14 \mathrm{~mm} \mathrm{Hg}$ at the inferior vena cava (IVC)-right atrium (RA) junction without thrombus, concerning for stricture. A radiograph of the chest best demonstrates the position of the AICD leads (Figure 1). Vascular surgery was unable to cross the lesion with a wire, therefore eliminating the possibility of endovascular angioplasty. A cardiac electrophysiologist, vascular surgeon, hepatobiliary surgeon, and cardiac surgeon concluded that open resection and reconstruction would be the best approach. The patient involved in this case provided informed consent for the study of the case and publication of a deidentified report.

In the operating room, the AICD generator was delivered and laser lead extraction performed. Access to the right

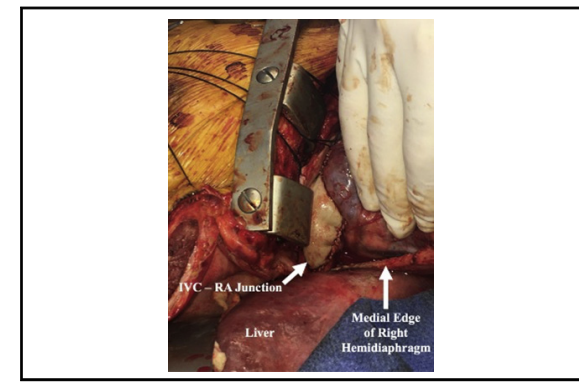

IVC-RA junction reconstruction via sternotomy extended into a right subcostal incision.

\section{CENTRAL MESSAGE \\ A symptomatic inferior vena cava-right atrial junction stenosis from AICD leads required a multidisciplinary team for reconstruction via midline ster- notomy extended under the right costal margin.}

See Commentaries on pages 34 and 36.

common femoral vein was obtained and a median sternotomy was performed and extended obliquely under the right costal margin. The liver was mobilized and the diaphragm was divided to expose the suprahepatic IVC. Next, the suprahepatic IVC-RA junction was mobilized and the oblique sinus was opened. Palpation of the IVC revealed a firm mass and the superior vena cava (SVC) had a chronic complete thrombosis.

Cardiopulmonary bypass was established with aortic and femoral venous cannulation. A right atriotomy revealed a lead extending from the SVC, through the RA, and making a $270^{\circ}$ turn around the IVC orifice before traversing through a perforation in the posterior leaflet of the tricuspid valve. The IVC-RA junction tissue was extremely fibrotic and scarred, leaving an opening of approximately $5 \mathrm{~mm}$. To control inflow from the IVC, we used a femoral IVC cannula that was withdrawn below the hepatic veins and applied vacuum assisted drainage. The lead was then divided in the RA and pulled from the left subclavicular 


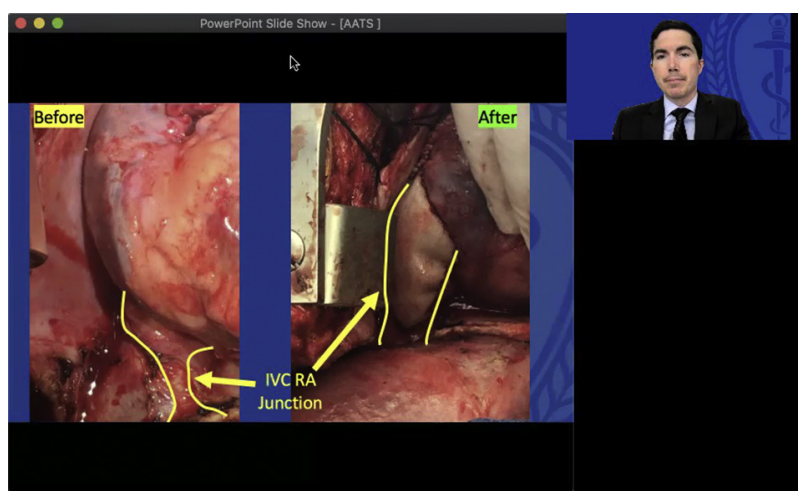

VIDEO 1. Case presentation at the 2021 AATS Cardiothoracic Resident case report competition. Video available at: https://www.jtcvs.org/article/ S2666-2507(21)00732-X/fulltext.

incision. We then dissected the lead from the apex of the right ventricle. A 3-mm perforation in the posterior tricuspid valve leaflet was closed with interrupted polypropylene suture. All leads were successfully extracted.

We then extended the right atriotomy across the right atrial/IVC junction through the stenosis and continued along the anterior aspect of the suprahepatic IVC. We resected $75 \%$ of the stenosis circumferentially. The IVC$\mathrm{RA}$ junction as well as the caudal portion of the RA was reconstructed with bovine pericardium and 5-0 polypropylene suture (Figure 2).

A postoperative transesophageal echocardiogram demonstrated mild tricuspid insufficiency and no flow acceleration into the RA from the IVC; the SVC remained occluded. The patient had an uneventful postoperative course and was discharged home from the hospital. Her

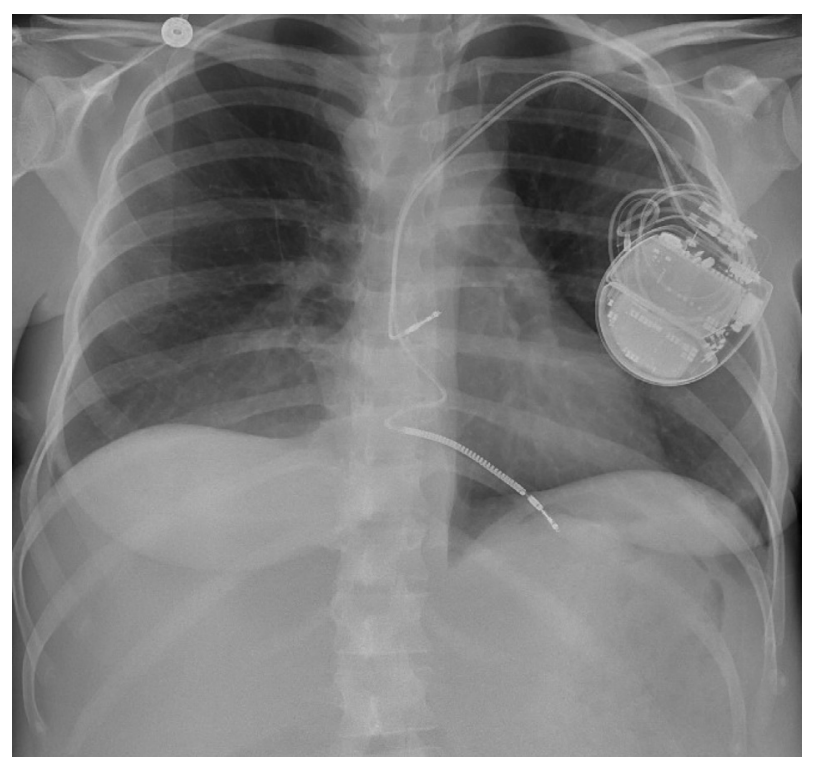

FIGURE 1. Preoperative radiograph of the chest demonstrating lead position for the automatic implantable cardioverter-defibrillator.

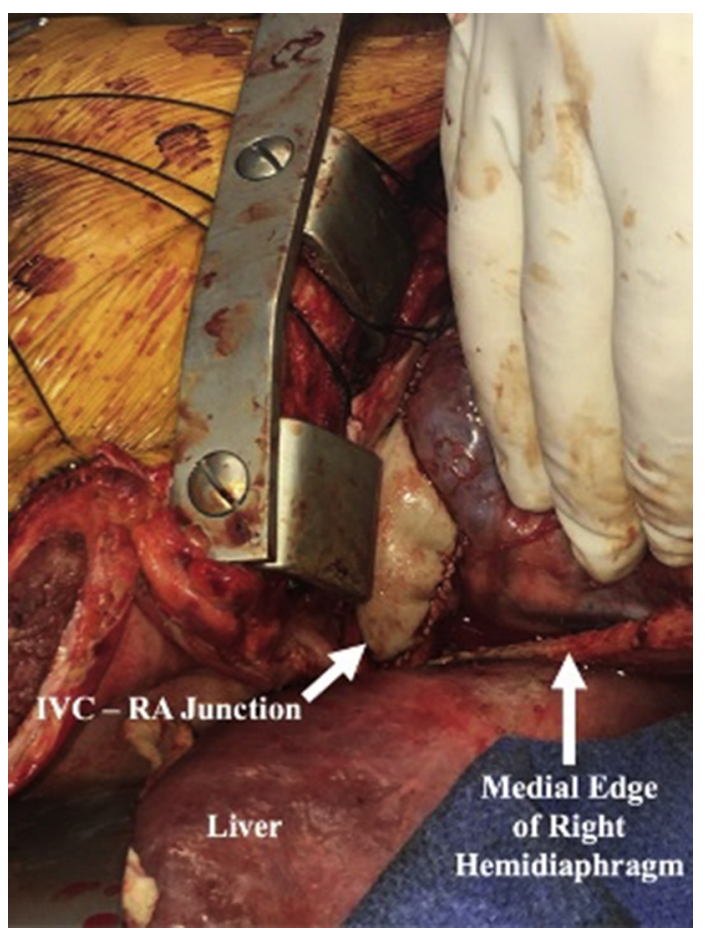

FIGURE 2. Inferior vena cava (IVC)-right atrium $(R A)$ junction reconstruction through sternotomy extended into a right subcostal incision.

preoperative symptoms completely resolved, and she had a significant improvement in function.

Venous thrombosis from implanted pacemakers or AICDs is thought to be due to inflammation induced by mechanical stress on the endothelium, leading to a cascade of thrombus formation, fibrosis, and stenosis. ${ }^{1}$ Asymptomatic venous stenosis is a known complication; $20 \%$ to $50 \%$ of these patients develop venous thrombosis, and incidence is greater with a greater number of leads and/or greater lead diameter. ${ }^{2}$ However, symptomatic venous stenosis only occurs in $1 \%$ to $3 \%$ of patients with implanted devices, and this has only been reported involving the SVC. ${ }^{1-3}$ Symptoms of SVC stenosis/occlusion represent SVC syndrome and include head and neck edema, shortness of breath, facial flushing, headache, dizziness, hand edema, and purple discoloration. ${ }^{4}$

Treatment of symptomatic stenosis involves a stepwise approach of anticoagulation, endovascular angioplasty, and lastly open surgery. Endovascular balloon angioplasty and stenting have been successful in treating stenosis or occlusion, but results in decreased patency when compared to patients undergoing procedures without device leads in situ.

The unique aspect of our case is the stenosis of the IVCRA junction combined with SVC occlusion. Due to extensive collateral circulation, the symptomatology of SVC syndrome did not present until the IVC became obstructed. The sequelae of portal hypertension, ascites, and abdominal venous distention were distinctive in this case and arose from nearly complete obstruction of venous return. Our 
approach illustrates that the common approach of patch venoplasty can be applied to the IVC-RA junction. For future cases, we recommend the use of a Hegar dilator to ensure a sufficient orifice for the IVC. We found a multidisciplinary team essential to planning and executing a surgical solution to this rare complication of AICD leads.

\section{References}

1. Desai D, Cooley R, Kress D, Allaqaband SQ. Surgical patch venoplasty after unsuccessful percutaneous balloon venoplasty for pacemaker lead-related superior vena cava stenosis. HeartRhythm Case Reps. 2018;4:141-3.
2. Abu-El-Haija B, Bhave PD, Campbell DN, Mazur A, Hodgson-Zingman DM Cotarlan V, et al. Venous stenosis after transvenous lead placement: a study of outcomes and risk factors in 212 consecutive patients. J Am Heart Assoc. 2015;4: e001878.

3. Park HW, Kim W, Cho JG, Kang JC. Multiple pacing lead-induced superior vena cava syndrome: successful treatment by balloon angioplasty. J Cardiovasc Electrophysiol. 2005;16:221-3.

4. Fu HX, Huang XM, Zhong L, Osborn MJ, Bjarnason H, Mulpuru S, et al. Outcome and management of pacemaker-induced superior vena cava syndrome. Pacing Clin Electrophysiol. 2014;37:1470-6.

5. Borsato GW, Rajan DK, Simons ME, Sniderman KW, Tan KT. Central venous stenosis associated with pacemaker leads: short-term results of endovascular interventions. J Vasc Interv Radiol. 2012;23:363-7. 